\title{
Electrolytes and glucose in cerebrospinal fluid of premature infants with intraventricular haemorrhage: role of potassium in cerebral infarction
}

\author{
P R STUTCHFIELD* AND R W I COOKE $\dagger$ \\ *Department of Child Health, Royal Liverpool Children's Hospital Alder Hey, and †Regional Neonatal Unit, \\ Liverpool Maternity Hospital
}

SUMmary Potassium, sodium, and glucose concentrations in cerebrospinal fluid and plasma were determined in 73 infants whose gestational ages ranged from 25 to 40 weeks. Six of 29 (21\%) neonates with intraventricular haemorrhages had raised potassium concentrations in the cerebrospinal fluid $(3 \cdot 7-30 \mathrm{mmol} / \mathrm{l})$; five developed cerebral infarctions. There was a significant correlation between sodium and glucose concentrations in plasma and cerebrospinal fluid with prolonged hypoglycorrhachia accompanying posthaemorrhagic hydrocephalus. Raised concentrations of potassium in cerebrospinal fluid occur with intraventricular haemorrhage and may contribute to the development of cerebral infarction.

The increased incidence of cerebral infarction in preterm infants with intraventricular haemorrhage has been well described ${ }^{1}$ and may result from common aetiological factors. There is, however, circumstantial evidence that intraventricular haemorrhage may lead to infarction. The median time of onset of cerebral infarction is reported to be significantly later than that of intraventricular haemorrhage. Cerebral vasospasm occurs after subarachnoid haemorrhage in adults and may lead to haemorrhagic infarction, thereby causing considerable morbidity and mortality. ${ }^{2} 3$ In premature infants with intraventricular and intracerebral haemorrhages, a pronounced reduction in regional cerebral blood flow has been shown on positron emission tomography. ${ }^{4}$

Vasoactive compounds are released after the lysis of red cells and platelets, and these are capable of causing vasospasm in vitro. Oxyhaemoglobin, prostaglandins, and lipid hydroperoxides have all been implicated in producing vasoconstriction of cerebral vessels. ${ }^{5}$ Edvinsson et al showed that high concentrations of potassium in the cerebrospinal fluid cause pronounced constriction of isolated human pial arterioles and small arteries. ${ }^{6}$ Concentrations sufficient to produce this effect were attained following lysis of blood clot in simulated cerebrospinal fluid. They proposed that high concentrations of potassium in the cerebrospinal fluid may play a part in the development of infarction accompanying intra- ventricular haemorrhage in neonates by inducing vasospasm in the surrounding cerebral tissue.

The aim of this study was to measure the concentrations of potassium in the cerebrospinal fluid of preterm infants who had had intraventricular haemorrhages, to determine whether high concentrations occur in vivo, and in addition to examine the effect of intraventricular haemorrhage on concentrations of sodium and glucose in the cerebrospinal fluid. The presence of ultrasound changes compatible with cerebral infarction was recorded.

\section{Patients and methods}

Neonates requiring lumbar puncture as part of a screen for infection were entered into the study. In addition to the cerebrospinal fluid obtained for culture, $0 \cdot 5-1 \mathrm{ml}$ was collected in a plain tube and centrifuged immediately to remove any intact red cells that may have been introduced by the trauma of the lumbar tap. The supernatant sodium, potassium, and glucose concentrations were measured in the cerebrospinal fluid as well as in heparinised and fluorinated blood samples obtained at the time of lumbar puncture. To minimise the risk of haemolysis, blood was obtained from a flushed umbilical arterial catheter or peripheral vein. The presence of haemolysis was reported by the laboratory staff when they had looked at the plasma.

Plasma and cerebrospinal fluid sodium and potas- 
sium concentrations were measured directly using ion selective electrodes (Corning 614). Potassium concentrations of greater than $10 \mathrm{mmol} / \mathrm{l}$ were determined by flame photometer (IL 543). Plasma and cerebrospinal fluid glucose concentrations were measured by the glucose oxidase oxygen consumption method (Beckman glucose analyser). The coefficient of variation for each of the above methods was $0.5 \%, 2 \%$, and $5 \%$, respectively.

Cranial ultrasound scans were carried out on preterm infants of less than 34 weeks' gestation daily for seven days, then one to three times/week, with a real time sector scanner with $7.5 \mathrm{mHz}$ transducer. More mature infants had ultrasound scans at the time of the lumbar puncture and as indicated thereafter. The patients were divided into groups according to the cranial ultrasound scan findings. Periventricular haemorrhage was graded according to the following simple classification: grade Isubependymal haemorrhage, grade II-intraventricular haemorrhage, and grade III-parenchymal echodensity adjacent to the ventricles.

Biochemical data within groups were analysed by the Pearson rank correlation test, and between groups by the Mann-Whitney $U$ test.

\section{Results}

Concentrations of potassium, sodium, and glucose in cerebrospinal fluid and plasma were measured on 68 occasions in 57 neonates aged 1-14 days, of 25-40 weeks' gestation. Thirty one samples of cerebrospinal fluid were also obtained from 28 neonates of 28-40 weeks' gestation (median 32), who had normal ultrasound scans. These constitute the control group. Ultrasound changes compatible with intraventricular haemorrhage grade I-III were present in 29 neonates of 25-34 weeks' gestation (median 28), who formed the intraventricular haemorrhage group. All echodense intracerebral lesions that were seen had occurred in association with intraventricular haemorrhages.

The cerebrospinal fluid potassium concentrations in patients in the control group ranged from $2 \cdot 8-3 \cdot 4$ $\mathrm{mmol} / \mathrm{l}$, mean (SD) $3 \cdot 1(0 \cdot 15) \mathrm{mmol} / \mathrm{l}$. There was no significant correlation between the cerebrospinal fluid and plasma potassium concentrations $(\mathrm{r}=0 \cdot 14)$ (fig 1); the cerebrospinal fluid potassium was maintained within a narrow range. Dividing the patients in the control group according to gestational ages of 25-29 weeks, 30-34 weeks, and 35-40 weeks, no significant differences were found in the cerebrospinal fluid potassium concentrations among the groups (mean (SD)) cerebrospinal fluid potassium concentration $3.15(0 \cdot 14) \mathrm{mmol} / \mathrm{l}, 3.09(0 \cdot 18)$, and $3.13(0 \cdot 1) \mathrm{mmol} / \mathrm{l}$, respectively) indicating that the

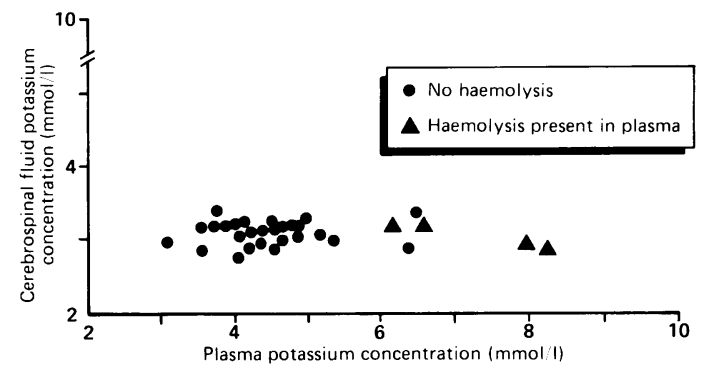

Fig 1 Cerebrospinal fluid and plasma potassium concentrations in neonates aged $<14$ days who did not develop intraventricular haemorrhages.

cerebrospinal fluid potassium concentration is as well controlled in the immature as in the mature infants.

The cerebrospinal fluid potassium concentration in the group with intraventricular haemorrhage ranged from 2.6 to $30 \mathrm{mmol} / \mathrm{l}$, mean (SD) $4.2(4.5)$ $\mathrm{mmol} / \mathrm{l}$. Six neonates had cerebrospinal fluid potassium concentrations two standard deviations above the mean in the control group, with potassium concentrations ranging from $3 \cdot 7-30 \mathrm{mmol} / \mathrm{l}$ (median 6.6) (fig 2 and table 1). Case 1 developed intracerebral echodense areas 24 hours after lumbar puncture. The intracerebral echodense areas in cases 1-3 became more extensive 24-96 hours after lumbar puncture.

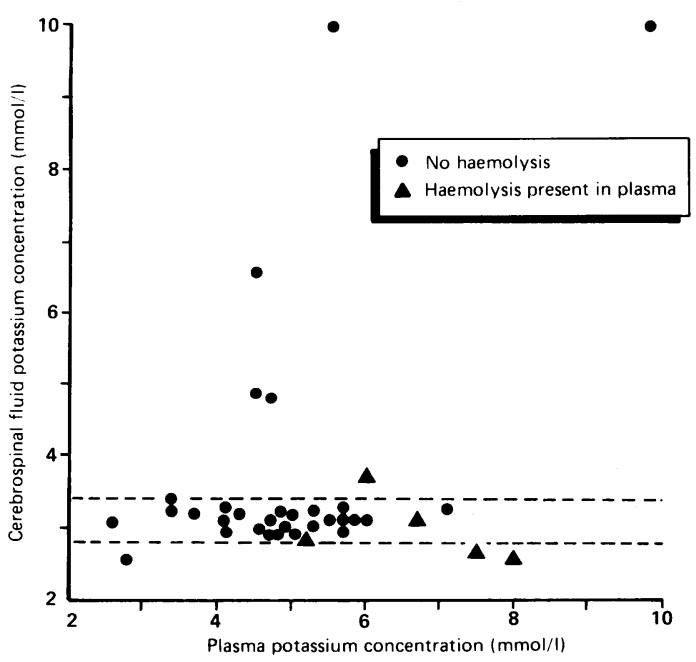

Fig 2 Cerebrospinal fluid and plasma potassium concentrations in neonates aged $<14$ days who had intraventricular haemorrhages. The two points at the top of the figure represent a cerebrospinal fluid potassium concentration of (left) $>10 \mathrm{mmol} / \mathrm{l}$ and (right) $30 \mathrm{mmol} / \mathrm{l}$. 
Table Details of six patients with intraventricular haemorrhage and cerebrospinal fluid potassium concentrations more than two standard deviations above the mean

\begin{tabular}{|c|c|c|c|c|}
\hline $\begin{array}{l}\text { Gestation } \\
\text { (weeks) }\end{array}$ & $\begin{array}{l}\text { Age } \\
\text { (days) }\end{array}$ & $\begin{array}{l}\text { Potassium } \\
\text { concentration } \\
\text { cerebro- } \\
\text { spinal } \\
\text { fluid } \\
(\text { mmol/l) }\end{array}$ & $\begin{array}{l}\text { Cranial } \\
\text { ultrasound } \\
\text { scan at } \\
\text { time of } \\
\text { lumbar } \\
\text { puncture }\end{array}$ & $\begin{array}{l}\text { Subsequent } \\
\text { ultrasound } \\
\text { appearance }\end{array}$ \\
\hline 27 & $<1$ & $4 \cdot 8$ & $\begin{array}{l}\text { Bilateral, } \\
\text { grade II }\end{array}$ & Porencephaly \\
\hline 28 & 1 & $6 \cdot 6$ & $\begin{array}{l}\text { Right, } \\
\text { grade III } \\
\text { Left, } \\
\text { grade II }\end{array}$ & $\begin{array}{l}\text { Hydrocephaly } \\
\text { Porencephaly }\end{array}$ \\
\hline 26 & 2 & 4.9 & $\begin{array}{l}\text { Left, } \\
\text { grade III }\end{array}$ & $\begin{array}{l}\text { Hydrocephaly } \\
\text { Porencephaly }\end{array}$ \\
\hline 25 & 4 & 30 & $\begin{array}{l}\text { Bilateral, } \\
\text { grade III }\end{array}$ & Died \\
\hline 25 & 6 & $3 \cdot 7$ & $\begin{array}{l}\text { Left, } \\
\text { grade III }\end{array}$ & Porencephaly \\
\hline 28 & 7 & $>10$ & $\begin{array}{l}\text { Bilateral, } \\
\text { grade II }\end{array}$ & Normal \\
\hline
\end{tabular}

Cerebrospinal fluid was obtained from the neonates with high cerebrospinal fluid potassium concentrations at the age of 1-7 days (median 2). Ultrasonographic changes of infarction were present from the ages of 1-5 days (median 2). A further six patients with intraventricular haemorrhage and ultrasonographic changes compatible with infarction had normal cerebrospinal fluid potassium concentrations at the time of cerebrospinal fluid sampling. The cerebrospinal fluid was, however, obtained later, at 1-13 days (median 10) with areas of intracerebral echodensities appearing from the ages of 2-4 days (median 2); high cerebrospinal fluid potassium concentrations may have occurred at an earlier stage. On follow up ultrasound scans all had developed intracerebral cystic changes, and the intracerebral echodensities were contiguous with the ventricular echodensity. These were compatible with ultrasound appearances of haemorrhagic infarction or extension of intraventricular haemorrhage. On follow up scans the intracerebral echodense areas had resolved with cystic formation confirming that destruction of brain tissue (infarcion) had occurred.

The mean (SD) cerebrospinal fluid sodium concentration in the control group was 134 (3) mmol/l and in the intraventricular haemorrhage group 133 (5.5) $\mathrm{mmol} / \mathrm{l}(\mathrm{p}=0.4)$ with a mean cerebrospinal fluid:plasma sodium ratio of 0.99 . There was a significant correlation between cerebrospinal fluid and plasma sodium in the two groups, $\mathrm{r}$ being 0.7 and 0.57 , respectively. The mean cerebrospinal fluid:plasma sodium ratio was $0.99(0.02)$ in the control group and $0.99(0.04)$ in the intraventricular haemorrhage group.

The cerebrospinal fluid glucose concentrations in the normal group (range $0 \cdot 5-8.1 \mathrm{mmol} / \mathrm{l}$, median 3 ) correlated significantly $(\mathrm{r}=0.7)$ with the plasma concentrations (range $0.5-10 \mathrm{mmol} / \mathrm{l}$, median 4.2 ) with a mean cerebrospinal fluid:plasma glucose ratio of $0 \cdot 8$ (range $0 \cdot 34-2 \cdot 6$ ). A significant correlation $(\mathrm{r}=0.8)$ was also found between cerebrospinal fluid glucose (range $0 \cdot 5-15 \cdot 1 \mathrm{mmol} / \mathrm{l}$, median $2 \cdot 8$ ) and plasma glucose (range 1.1-27 mmol/l, median 4.4) concentrations in the intraventricular haemorrhage group, with a mean cerebrospinal fluid:plasma glucose ratio of 0.6 (range $0.08-2$ ). The lowest ratios were associated with the more extensive haemorrhages. Culture of cerebrospinal fluid was negative in all cases.

Thirteen samples of cerebrospinal fluid were obtained from 10 patients aged 15-58 days (gestation 25-28 weeks) who developed posthaemorrhagic hydrocephalus. Cerebrospinal fluid was removed at intervals before insertion of a ventriculoperitoneal shunt. Cultures of cerebrospinal fluid were negative in all cases. The cerebrospinal fluid glucose concentrations were significantly lower $(p<0.001)$ in this group of patients than in the control or intraventricular haemorrhage groups, with a mean cerebrospinal fluid:plasma glucose ratio of 0.32 , range $0 \cdot 13-0 \cdot 6$ (fig 3). Two infants had raised cerebrospinal fluid potassium concentrations. One baby aged 17 days (gestation 28 weeks) had a large intraventricular clot with cerebral infarction on ultrasound scan. The ventricular cerebrospinal fluid potassium concentration was more than $10 \mathrm{mmol} / \mathrm{l}$. Unfortunately the actual concentration was not determined. A baby of 26 weeks' gestation aged 58 days developed a haemorrhagic infarction in an area of porencephaly after repeated ventricular taps for non-communicating hydrocephalus. The ventricular cerebrospinal fluid potassium concentration was $7 \cdot 6$ $\mathrm{mmol} / \mathrm{l}$.

Cerebrospinal fluid was obtained from a further six patients aged 14-102 days (gestation 25-35 weeks) at the time of infection screen. All had normal cranial ultrasound scans throughout. The cerebrospinal fluid:plasma glucose ratio in this group ranged from 0.4 to 1.2 , mean $0.7(0.2)$. All but one had cerebrospinal fluid potassium concentrations within the range of the control group. On day 27 one infant of 25 weeks' gestation had a cerebrospinal fluid potassium concentration of $9 \mathrm{mmol} / \mathrm{l}$ (plasma concentration 5.3), and on day 34 a cerebrospinal fluid concentration of $4.3 \mathrm{mmol}$. 


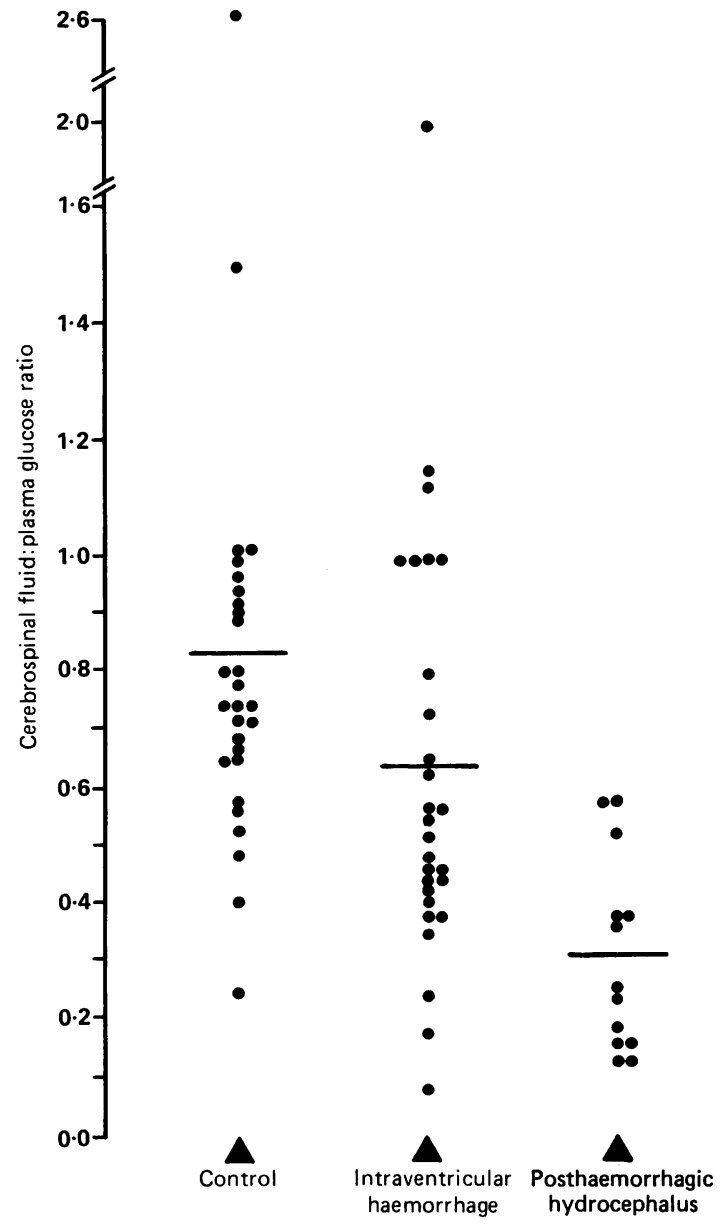

Fig 3 Ratio of cerebrospinal fluid:plasma glucose in the three groups of babies.

The sodium cerebrospinal fluid:plasma ratio was $0.98(0.04)$ and $0.99(0.008)$ in the posthaemorrhagic hydrocephalus group and the last group without intraventricular haemorrhage, respectively.

\section{Discussion}

Cerebrospinal fluid potassium concentration in adults and various animals (for example, rabbits, cats, dogs, and goats) is controlled within the narrow range $2 \cdot 5-3 \cdot 3 \mathrm{mmol} / \mathrm{l}$ despite wide variations in plasma potassium concentrations. ${ }^{78}$ There is evidence that the maintenance of the cerebrospinal fluid potassium, with increased efflux of this ion in the presence of raised concentrations, depends on an active sodium/potassium pump. ${ }^{8}$ This control is maintained despite the association of various dis- eases (for example, tuberculosis, meningitis, hypertension, and encephalopathy). Cooper et al did not find alterations in cerebrospinal fluid potassium concentrations in adult patients with cerebral haemorrhages despite the presence of large amounts of blood in the subarachnoid space. ${ }^{9}$ We found that the mechanisms for maintaining cerebrospinal fluid potassium concentrations are established in neonates even at 26 weeks' gestation, the cerebrospinal fluid potassium ranging from $2 \cdot 8-3 \cdot 4 \mathrm{mmol} / \mathrm{l}$ irrespective of the plasma concentration. In the presence of intraventricular blood, however, raised cerebrospinal fluid potassium concentrations were found.

Six of 30 of the neonates $(20 \%)$ aged less than 14 days who had intraventricular haemorrhages on ultrasound scan had cerebrospinal fluid potassium concentrations two standard deviations outside the mean for our control population (median $6.6 \mathrm{mmol} / \mathrm{l}$, range 3.7-30). The cerebrospinal fluid potassium concentrations were recorded mainly in specimens obtained at lumbar puncture. These concentrations have been shown to be lower than ventricular concentrations. The most relevant cerebrospinal fluid potassium concentration is that measured adjacent to the ventricular blood clot at the site of release from haemolysing red cells. This study was limited, as cerebrospinal fluid was only obtained when there was a clinical indication for lumbar puncture. Ideally the cerebrospinal fluid concentrations should be measured daily to detect any transient rise that may occur with peak haemolysis. After the addition of blood to simulated cerebrospinal fluid in vitro in concentrations of 10,30 , and $50 \%$, peak cerebrospinal fluid potassium concentrations 6,16 , and $24 \mathrm{mmol} / \mathrm{l}$ were obtained, respectively by the fourth day. ${ }^{6}$ With small intraventricular haemorrhages the homoeostatic mechanisms concerned in the control of the cerebrospinal fluid potassium concentration may be adequate, but with large volumes these may be overwhelmed leading to high circulating cerebrospinal fluid potassium concentrations that are detectable by lumbar puncture.

Five of 11 patients $(45 \%)$ who had evidence of cerebral infarction on ultrasound scans had raised cerebrospinal fluid potassium concentrations. These did not represent the peak concentrations achieved, but are random samples as already discussed. A raised cerebrospinal fluid potassium concentration was recorded in only one patient with grade II periventricular haemorrhage 24 hours before the appearance of intracerebral echodensity adjacent to and contiguous with the ventricle. The other four infants already had grade III periventricular haemorrhages at the time of their lumbar punctures. Intracerebral echodensity was not recorded in the 
baby of 28 weeks' gestation who had a cerebrospinal fluid potassium concentrations of more than 10 $\mathrm{mmol} / \mathrm{l}$ at 7 days.

The intracerebral echodense areas represent either haemorrhage into infarcted tissue or venous infarction secondary to haemorrhage, as described by Gould $e t$ al. ${ }^{10}$ The presence of infarction is supported by the development of cystic lesions in those who survived.

Two further patients with posthaemorrhagic hydrocephalus had raised potassium concentrations, one of whom had had a recent haemorrhage into an area of infarct. Edvinsson et al showed that there was pronounced vasoconstriction of pial and cerebral blood vessels when potassium concentrations were greater than $10 \mathrm{mmol} / \mathrm{l} .^{6}$ They proposed that potassium affects vascular smooth muscle through the depolarisation of the cell membrane and the opening of potential operated calcium channels. High concentrations of extracellular potassium result in a large increase in cerebral glucose utilisation associated with a spread of intense neuronal activity followed by membrane depolarisation and electrical depression. ${ }^{11}$ Edvinsson et al concluded that a high cerebrospinal fluid potassium concentration produced by haemolysing red cells from an intraventricular haemorrhage may induce vasospasm in the surrounding cerebral tissue in addition to increasing its energy needs. In this way intraventricular haemorrhage may lead to cerebral infarction.

In our study a raised cerebrospinal fluid potassium concentration was present in one patient with intraventricular haemorrhage before the appearance of echodensity in the cerebral tissue. Evidence of cerebral infarction or haemorrhage, or both, was already present at the time of the lumbar punctures in the other neonates who had raised cerebrospinal fluid potassium concentrations. Within the limitations of this study we did not demonstrate that raised cerebrospinal fluid potassium concentrations lead to cerebral infarction, but did find that raised cerebrospinal fluid potassium concentrations do occur in premature infants with intraventricular haemorrhage in contrast with adults, ${ }^{9}$ and the possibility remains that this may contribute to the development of infarction.

Extracellular potassium plays an important part in the regulation of neuronal excitability and it can therefore be expected that small changes in the cerebrospinal fluid potassium concentration may produce profound changes in the neurological and clinical state. The irritability and hypertonicity seen in some infants with intraventricular haemorrhage may be produced in this way, but we did not study this.
The cerebrospinal fluid sodium concentrations correlated with those in plasma, maintaining an osmotic equilibrium even in the presence of an intraventricular haemorrhage. Cooper et al, however, found a significant increase in the cerebrospinal fluid sodium concentrations in adult patients who had had cerebral haemorrhage. ${ }^{9}$

The cerebrospinal fluid glucose concentrations correlated with those in plasma, with a mean cerebrospinal fluid:plasma glucose ratio of $0 \cdot 8$ in the control group A cerebrospinal fluid:plasma glucose ratio of 0.4 represents the lower limit of normal. ${ }^{12}$ Babies with the changes of extensive haemorrhage had the lowest ratios and the cerebrospinal fluid concentrations of glucose were low. For those who developed hydrocephalus the ratio remained low, with low white cell counts for up to 57 days although on ultrasound scan the blood clot had resolved. The reported gradual development of low cerebrospinal fluid glucose concentrations that reaches a trough when the number of viable cells in the cerebrospinal fluid is low with a slow return to normal, ${ }^{13}$ supports the hypothesis that alteration or inhibition of the glucose transport system occurs by breakdown products of blood. Hypoglycorrhachia may persist for weeks, as shown in our study. No association has been found between the severity of intraventricular haemorrhage and the persistence of blood on computed tomography, the development of hydrocephalus, ${ }^{14}$ or neurological outcome. ${ }^{15}$

The mechanisms of control of cerebrospinal fluid electrolytes and glucose are present in the most immature infant but may be disturbed by intraventricular haemorrhage. When intraventricular blood haemolyses, high cerebrospinal fluid potassium concentrations do occur, and may contribute to the development of cerebral infarction as a result of vasospasm.

We thank Dr A Stock, Dr P Baines, and Dr I Blakebrough for their help with this study, and Mrs A Longworth for typing the manuscript.

\section{References}

1 Sinha SK, Davics JM, Sims DG, Chiswick ML. Rclation between periventricular haemorrhage and ischacmic brain lesions diagnosed by ultrasound in very preterm infants. Lancet 1985;ii:1154-6.

2 Fisher CM, Kistler JP, Davis JM. Relation of cerebral vasospasm to subarachnoid haemorrhage visualised by computerised tomographic scanning. Neurosurgery 1980;6:1-8.

${ }^{3}$ Saito I, Ueda Y, Sano K. Significance of vasospasm in the treatment of ruptured intracranial aneurysms. J Neurosurg 1977;47:412-29.

4 Volpe JJ, Herscovitch P, Perlman JM, Raichle ME. Positron emission tomography in the newborn: extensive impairment of regional cerebral blood flow with intraventricular haemorrhage 
and hacmorrhagic intracerebral involvement. Pediatrics 1983; 72:589-601.

${ }^{5}$ Sasaki T, Asano T, Takakura K, Sano K, Kassell NF. Nature of the vasoactive substance in the CSF from patients with subarachnoid hacmorrhage. J eurosurg 1984;60:1186-91.

${ }^{6}$ Edvinsson L, Lou HC, Tvede K. On the pathogenesis of regional cerebral ischemia in intracranial hemorrhage: a causal influence of potassium? Pediatr Res 1986;20:478-80.

${ }^{7}$ Bradbury MWB, Stubbs J, Hughes IE, Parker P. The distribution of potassium, sodium, chloride and urea between lumbar cerebrospinal fluid and blood scrum in human subjects. Clin Sci 1963;25:97-105.

${ }^{*}$ Katzman R, Pappius HM. Brain electrolytes and fluid metabolism. Baltimore: Williams and Wilkins, 1973:80-95.

${ }^{9}$ Cooper ES, Lechner E, Bellet S. Relation between serum and cerebrospinal fluid electrolytes under normal and abnormal conditions. Am J Med 1955;18:613-21.

10 Gould SJ, Howard S, Hope PL, Reynolds EOR. Periventricular intraparenchymal cerebral haemorrhage in preterm infants: the role of venous infarction. J Pathol 1987;151:197-202.

"Shinohara M, Dollinger B, Brown G, Rapoport S, Sokoloff L. Cerebral glucose utilisation: local changes during and after recovery from spreading cortical depression. Science 1979;203: 188-90.

12 Donald PR, Malan C, Van der Walt A. Simultaneous determination of cerebrospinal fluid glucose and blood glucose concentrations in the diagnosis of bacterial meningitis. $J$ Pediatr 1983;103:413-5.

13 Mathew OP, Bland HE, Pickens JM, James EJP. Hypoglycorrhachia in the survivors of neonatal intracranial haemorrhage. Pediatrics 1979:63:851-4.

14 Mathew OP, Volpe JJ. Neonatal intraventricular haemorrhage: hypoglycorrhachia and its relationship to CSF lactate levels. $J$ Pediatr 1980;97:292-5.

15 Th Deonna A, Calame A, Van Melle G, Prod'hom S. Hypoglycorrhachia in neonatal intracranial haemorrhage. Relationship to post haemorrhagic hydrocephalus. Helv Paediatr Acta 1977;32:351-61.

Correspondence to Dr PR Stutchfield, Glan Clwyd Hospital, Bodelwyddan, Rhyl, Clwyd LL18 5UJ.

Accepted 1 December 1988 